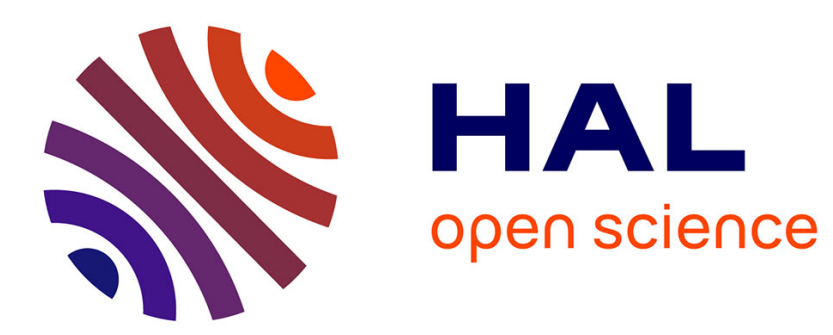

\title{
L'analyse du financement de l'entreprise selon la théorie des options
}

\author{
Nathalie Mourgues
}

\section{To cite this version:}

Nathalie Mourgues. L'analyse du financement de l'entreprise selon la théorie des options. Les cadres conceptuels, May 1991, France. pp.cd-rom. hal-00823333

\section{HAL Id: hal-00823333 \\ https://hal.science/hal-00823333}

Submitted on 18 Sep 2014

HAL is a multi-disciplinary open access archive for the deposit and dissemination of scientific research documents, whether they are published or not. The documents may come from teaching and research institutions in France or abroad, or from public or private research centers.
L'archive ouverte pluridisciplinaire HAL, est destinée au dépôt et à la diffusion de documents scientifiques de niveau recherche, publiés ou non, émanant des établissements d'enseignement et de recherche français ou étrangers, des laboratoires publics ou privés. 


\section{L'offre et la demande de l'information comptable}

Coordinateurs : Michel LEVASSEUR, Université de Lille, Raymond MAEDER, Groupe HEC

"L'analyse du financement de l'entreprise selon la théorie des options"

Nathalie MOURGUES, Université d'Orleans, Instiłut Orléanais de Finance 



\title{
INSTITUT ORLÉANAIS DE FINANCE
}

ÉQUIPE DE RECHERCHE ASSOCIÉE AU C.N.R.S.

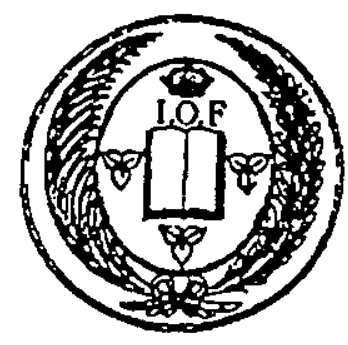

\section{L'ANALYSE DU FINANCEMENT}

\section{DE L'ENTREPRISE}

\section{SELON LA THÉORIE DES OPTIONS}

\author{
N. MOURGUES
}

UNIVERSITÉ D'ORLÉANS

FACULTÉ DE DROIT, D'ÉCONOMIE ET DE GESTION 


\section{Sommaire}

Introduction

1. Une représentation du bilan de l'entreprise selon la théorie des options 1

11. Modèle d'entreprise simulée …...................................................... 2

12. La nature optionnelle des sources de financement ................................ 4

2. L'évaluation de la dette risquée .......................................................... 8

21. Les déterminants de la valeur des sources de financement ......................... 8

22. Une simulation chiffrée ............................................................ 10

3. La formation de la structure financlère .............................................. 14

31. Structure financière et partage du risque ............................................ 14

32. Structure financière et risque du projet dinvestissement ........................ 15

3 3. Les garanties prises par les créanciers …........................................ 17

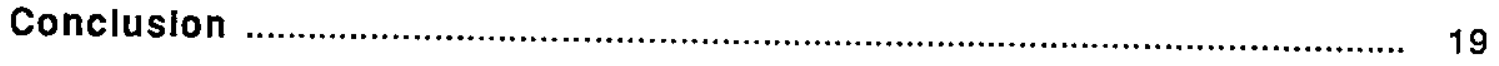

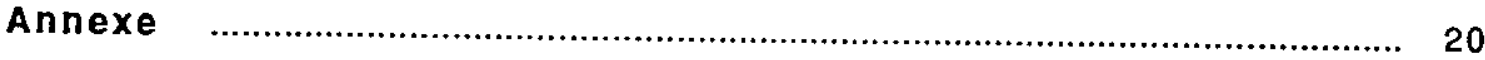

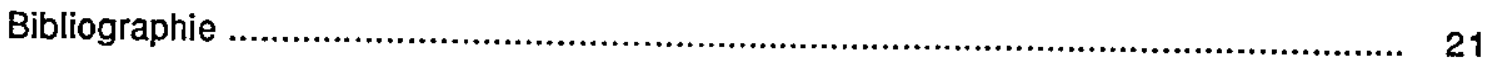





\section{Introduction}

Comme l'indique R. Cobbaut (1987), "de nombreuses pratiques financières constituent en réalité des opérations sur options ou peuvent, en tout cas, être analysées comme telles"; et l'auteur d'ajouter : "en réalité, c'est l'ensemble des décisions de financement qui peuvent être traitées comme des opérations sur options"1. Ces propos résument la position prise par certains auteurs pour analyser les problèmes de financement de l'entreprise.

L'objet du présent article est de montrer l'intérêt que présente l'application de la théorie des options pour analyser le financement de l'entreprise et l'équilibre financier entre actionnaires et créanciers. En cela notre objectif est partiellement didactique ; en même temps nous espérons bien mettre en évidence quelques résultats importants quant à l'évaluation des sources de financement et le choix d'une structure financière.

L'article est présenté en trois sections. Dans une première section nous donnons une représentation du bilan de l'entreprise selon la théorie des options. Pour ce faire, nous avons choisi de simuler une situation très simple d'entreprise afin d'avoir une lecture aisée de son financement en termes d'options. Dans la deuxième section nous traitons de l'évaluation de la dette risquée. C'est là un des apports importants de l'approche que de permettre non seulement l'évaluation des capitaux propres de l'entreprise mais aussi celle de la dette risquée à travers l'évaluation de l'option de défaillance qui s'y rattache. Enfin, dans une troisième section, nous posons le problème du choix de la structure financière de l'entreprise, au regard de trois éléments : le risque du projet financé, l'acceptation, ou le refus, des créanciers de participer à la prise de risque et les garanties qui peuvent être négociées entre les deux catégories d'agents.

\section{Une représentation du bilan de l'entre- prise selon la théorie des options}

Afin de présenter, selon la théorie des options, la vision de la structure financière de l'entreprise, nous envisageons une situation d'entreprise la plus simple qu'il soit. Après avoir présenté cette situation (11), nous décrivons en termes d'options le financement de l'entreprise simulée (12).

$1_{\text {pp. 349-50. }}$ 


\section{Modèle d'entreprise simulée}

Considérons la création d'une entreprise réalisée en vue de l'exploitation d'un projet industriel d'une durée de $n$ périodes. Le projet doit rapporter des flux annuels de liquidités de $C_{t}(t=1,2, \ldots n)$ pour un montant d'investissement, en $t=0$, de $V_{0}$. Le taux de rentabilité requis, compte tenu du risque du projet, est de $R_{\theta}$. Le risque de ne pas recevoir le taux de rentabilité est mesuré par la variance de $R_{e}$, soit $\sigma^{2}$. L'espérance de la valeur actualisée nette du projet au taux requis est nulle. Autrement dit, la valeur actualisée des flux attendus est égale au montant investi. On suppose, par ailleurs, que les flux attendus de trésorerie sont réinvestis au taux $R_{\theta}$; il n'y a, donc, pas de distribution de dividendes. Le projet consiste, ainsi, à échanger une valeur $V_{0}$ en $t=0$ contre une espérance de valeur $V_{n}$ en $t=n$ sachant que :

$$
\begin{aligned}
V_{0} & =\sum_{t=1}^{n} c_{t}\left(1+R_{e}\right)^{t} \\
& V_{n}=\sum_{t=1}^{n} c_{t}\left(1+R_{e}\right)^{n t t} \\
\text { avec } \quad V_{0} & =V_{n}\left(1+R_{e}\right)^{-n}
\end{aligned}
$$

Le projet peut être financé par actions ordinaires $\left(C_{0}\right)$ et par dette $\left(D_{0}\right)$. Le taux d'intérêt de la dette négociée par les actionnaires auprès des créanciers est de $R_{i}$. Le remboursement de la dette doit intervenir à la fin de la nième année, il n'y a pas de coupon annuel, la rémunération des créanciers résultant de la différence entre la valeur de remboursement prévue de la dette $\left(D_{n}\right)$ et la valeur d'émission $\left(D_{0}\right){ }^{1}$

Le rapport "dette/capitaux propres" n'est pas défini a priori dans son montant : la fixation de ce rapport est une résultante des accords contractuels entre actionnaires et créanciers ; nous verrons ci-après qu'il dépend de la participation éventuelle des créanciers au partage du risque du projet d'investissement financé.

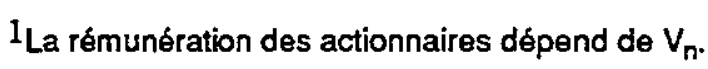


Le bilan, valeurs de marché, de l'entreprise, en $t=0$, est donc le suivant :

\begin{tabular}{|c|c|}
\hline $\begin{array}{l}\text { VALEUR DE L'ACTIF } \\
=V_{0}\end{array}$ & $\begin{array}{l}\text { VALEUR DES } \\
\text { CAPITAUX PROPRES } \\
=C_{0} \\
=V_{0}-D_{0}\end{array}$ \\
\hline$=\sum_{t=1}^{n} C_{t}\left(1+R_{e}\right)^{t}$ & $\begin{array}{l}\text { VALEUR DE LA DETTE } \\
\qquad=D_{0} \\
\quad=D_{n}\left(1+R_{i}\right)^{-n}\end{array}$ \\
\hline
\end{tabular}

A l'issue du projet, en $\mathrm{t}=\mathrm{n}$, il est prévu que l'entreprise soit dissoute. A ce moment-là, les actionnaires payent la valeur de remboursement de la dette $\left(D_{n}\right)$ et s'attribuent la valeur résiduelle de l'actif, soit $V_{n}$ la valeur, en $n$, de cet actif et $C_{n}$ la valeur des capitaux propres correspondante. La valeur des capitaux propres $\left(C_{n)}\right.$ est donc égale à la différence entre la valeur terminale de l'actif $\left(V_{n}\right)$ et la valeur de remboursement de la dette $\left(D_{n}\right)$.

Enfin, pour terminer, on posera que l'entreprise est organisée juridiquement de sorte que la responsabilité juridique des actionnaires à l'égard des créanciers est limitée à leur apport à la société1.

A la période $n$, quelle sera la situation patrimoniale des créanciers et des actionnaires ?

La richesse des actionnaires va osciller entre 0 et $\left(V_{n}-D_{n}\right)$ :

$$
C_{n}=\max \left[O,\left(V_{n}-D_{n}\right)\right]
$$

Elle sera de 0 si la valeur de l'entreprise est égale ou inférieure à $D_{n}$ et de $\left(V_{n}-D_{n}\right)$ si $V_{n}$ est supérieure à $D_{n}$.

La richesse des créanciers sera comprise entre $V_{n}$ et $D_{n}$ :

$$
D_{n}=\min \left[V_{n}, D_{n}\right]
$$

Elle sera de $D_{n}$ si la valeur de l'actif est égale ou supérieure à $V_{n}$, sinon elle sera de $V_{n}$, sachant que $V_{n}$ peut être nulle.

${ }^{1}$ Cas par définition des SA et des SARL. 


\section{La nature optionnelle des sources de financement}

Les caractéristiques de l'entreprise étant posées, la relation actionnaires-créanciers peut être décrite en termes d'options.

A l'échéance, selon la valeur de la dette par rapport à la valeur des actifs, on peut observer deux cas de figure symétriques : la première si $\left(V_{n}-D_{n}\right)>0$ et la seconde si $\left(V_{n}-D_{n}\right) \leq 0$.

$\left(V_{n}-D_{n}\right)>0$ :

Si la valeur des actifs est supérieure à la valeur de la dette, l'actionnaire fait valoir ses droits sur la valeur de l'actif, savoir : il rembourse les créanciers et s'approprie la valeur résiduelle $\left(V_{n}-D_{n}\right)$.

Dans ce cas de figure, tout se passe comme si l'actionnaire était détenteur d'une option d'achat sur la valeur de l'actif : option à exercer auprès des créanciers, avec pour valeur d'exercice le montant de la dette et pour date d'exercice l'échéance de la dette.

Ce premier cas de figure conduit à une première lecture ou représentation du rapport actionnaires-créanciers dans le financement de l'entreprise. Cette lecture est la suivante. L'actionnaire a le pouvoir de décision et de contrôle. A l'initiation du projet il transfère aux créanciers l'actif de la société. En contrepartie, il reçoit une certaine source de financement, $D_{0}$, de la part des créanciers. Par ailleurs, il paye une option d'achat, $\mathrm{C}_{0}$, sur l'actif, option qu'il pourra exercer à l'échéance de la dette si la valeur de cet actif (le sous-jacent) est supérieure à la valeur de remboursement de la dette (ou prix d'exercice de l'option).

\section{$\left(V_{n}-D_{n}\right) \leq 0:$}

Si la valeur de l'actif est inférieure ou égale à $D_{n}$, la valeur de l'option d'achat devient nulle, l'actionnaire abandonne la valeur de l'actif aux créanciers, ces derniers sont remboursés à concurrence du montant correspondant.

Dans ce second cas de figure, tout se passe comme si l'actionnaire était détenteur d'une option de vente, à l'égard des créanciers, sur l'actif de la société ; option lui donnant le droit de vendre l'actif aux créanciers en contrepartie et cela à un prix et une date d'exercice fixés respectivement à la valeur et à l'échéance de la dette. 
Dans ce cas de figure nous avons une deuxième représentation ${ }^{1}$ du rapport actionnaires-créanciers que voici. A l'initiation du projet d'investissement, l'actionnaire qui a le pouvoir de décision et de contrôle acquiert l'actif. Pour ce faire, l'actionnaire réalise une double opération avec les créanciers. D'une part, il emprunte un montant de ressources nécessaires au taux sans risque, d'autre part, il achète une option de vente sur l'actif. A l'échéance l'actionnaire, acheteur d'un "put", exercera son option si la valeur de l'actif est inférieure à la valeur de remboursement de la dette. ${ }^{2}$

En définitive, c'est grâce à cette option de vente que l'actionnaire a la possibilité de céder les actifs à la valeur de la dette et ce quand bien même la valeur des actifs est inférieure au montant à rembourser aux créanciers. On observera, à l'évidence, que si $V_{n}<D_{n}$, l'entreprise est en situation de faillite. En conséquence, la valeur de l'option de vente représente la valeur économique de la responsabillté IImitée des actionnalres à l'égard des créanclers.

On observera la parfaite convergence des deux interprétations de la relation actionnaires-créanciers que nous venons de voir. Dans la première représentation l'actionnaire est détenteur d'une option d'achat. Ce "call" n'est autre qu'une option synthétique correspondant au deuxième cas de figure dans lequel l'actionnaire est en possession de l'actif sous-jacent et d'une option de vente, option de vente et option d'achat ayant la même échéance et la même valeur d'exercice.

Autrement dit, on observe bien la stricte équivalence qui doit exister, selon la théorie des options, entre les deux situations suivantes:

- la situation de détention d'un actif sous-jacent, ici la détention de l'actif de l'entreprise par l'actionnaire, ${ }^{3}$

- et la situation de détention d'options sur cet actif, détention simultanément d'un "call" et d'un "put" ayant même date et même valeur d'exercice.

En définitive, à l'échéance, les équations suivantes se vérifient:

$$
\begin{aligned}
& \text { Si } V_{n}>D_{n} \\
& \text { - l'option d'achat est }>0 \\
& \text { - l'option de vente est }=0 \\
& \Leftrightarrow \quad \text { valeur de l'option d'achat }\left(C_{n}\right) \\
& \text { + valeur de la dette }\left(D_{n}\right) \\
& =\text { valeur de l'actif } \quad\left(V_{n}\right)
\end{aligned}
$$

\footnotetext{
${ }^{1}$ Cette deuxième représentation est strictement complémentaire, en termes optionnels, de la première pour expliquer le dénouement de l'accord contractuel actionnaires-créanciers.

2On remarquera que le prix de loption de vente est contenu dans la valeur d'émission de la dette pour une valeur de remboursement donnée (cf. ci-après).

${ }^{3}$ Situation qui correspond d'ailleurs à la vision traditionnelle de l'actionnaire propriétaire de l'entreprise.
} 
- SI $V_{n} \leq D_{n}$

- l'option d'achat est $=0$

- l'option de vente est $>0$

$\Leftrightarrow \quad$ valeur de la dette $\left(D_{n}\right)$

= valeur de l'actif $\left(V_{n}\right)$

+ valeur de l'option de vente $\left(P_{n}\right)$

A ce stade de la description du financement de l'entreprise, on peut reformuler le bilan de la société en valeur de marché, en $\mathrm{t}=0$. Cette reformulation permet d'exprimer en termes bilantiels la valeur de l'option de vente et, par voie de conséquence, la valeur de la dette risquée ainsi que la valeur de l'option d'achat.

On rappellera que :

$V_{0}=$ valeur de l'actif en $t=0$

$C_{0}=$ valeur des capitaux propres, ou encore valeur de l'option d'achat sur les actifs, en $\mathrm{t}=0$

Soit par ailleurs :

$P_{0}=$ la valeur de l'option de vente en $t=0$

$R_{F}=$ le taux de la dette.sans risque

$D_{0}^{\prime}=$ la valeur actuelle de $D_{n}$ (= valeur de remboursement de la dette ou prix d'exercice des options) actualisee au taux sans risque

$=D_{n}\left(1+B_{F}\right)^{-n}$

$R_{1}=$ le taux de la dette risquée

$D_{0}=$ la valeur actuelle de $D_{n}$ au taux $R_{i}$

$=D_{n}\left(1+R_{i}\right)^{n}$

On peut écrire l'égalité :

$$
C_{0}+D_{0}^{\prime}=V_{0}+P_{0}
$$

c'est-à-dire :

valeur de l'option d'achat sur les actifs de l'entreprise

+ valeur de la dette au taux sans risque

= valeur des actifs de l'entreprise

+ valeur de l'option de vente 
L'égalité (1) est équivalente à l'égalité (2) ci-après :

$$
C_{0}+D_{0}=V_{0}
$$

c'est-à-dire :

valeur de l'option d'achat

+ valeur de la dette risquée

= valeur de l'entreprise

Cette équation n'exprime pas autre chose que le bilan de l'entreprise en valeur de marché en $\mathrm{t}=0$.

\begin{tabular}{|c|c|}
\hline $\begin{array}{l}\text { VALEUR ECONOMIQUE } \\
\text { DE L'ENTREPRISE } \\
=V_{0}\end{array}$ & $\begin{aligned} & \text { VALEUR DES } \\
& \text { CAPTAUUX PROPRES } \\
&=C_{0} \\
&=V_{0}-D_{0} \\
&=V_{0}-\left(D_{0}^{\prime}+P_{0}\right)\end{aligned}$ \\
\hline$=\sum_{t=1}^{n} C_{t}\left(t+R_{e}\right)^{t}$ & $\begin{array}{l}\text { VALEUR DE LA DETTE } \\
=D_{0} \\
=D_{n}\left(1+R_{F}\right)^{-n}-P_{0} \\
=D_{n}\left(1+R_{i}\right)^{-n}\end{array}$ \\
\hline
\end{tabular}

Ainsi, pour une valeur négociée du remboursement de la dette on a :

$$
D_{0}=D_{n}\left(1+R_{i}\right)^{-n}
$$

Cette expression est équivalente à :

$$
D_{0}=D_{n}\left(1+R_{F}\right)^{-n}-P_{0}
$$

II en résulte, pour une valeur d'actif, une valeur de capitaux propres de :

$$
\begin{aligned}
C_{0} & =V_{0}-D_{0} \\
& =V_{0}-\left[D_{0}^{\prime}+P_{0}\right]
\end{aligned}
$$

avec $D_{0}^{\prime}=D_{n}\left(1+R_{F}\right)^{-n}$ 
Cette description bilantielle de l'entreprise présente de nombreux intérêts comme les sections suivantes le montreront. Elle permet, d'abord, d'analyser le prix de l'optlon de défalllance de l'entreprise et par là même elle permet de mesurer la prime de risque à offrir aux créanciers dans l'hypothèse oủ ces derniers acceptent de participer au partage du risque relatif au projet financé. Elle permet, ensuite, d'analyser la structure financlère de l'entreprise selon que les créanciers prennent une part plus ou moins importante dans la fonction de prise du risque. Par ailleurs, la présentation en termes d'options est sous-jacente dans tous modèles d'analyse des décisions financières lorsqu'on prend en compte les conflits d'intérêts entre actionnaires-créanciers liés à la relation d'agence existant entre ces agents.

\section{ש. 2. L'évaluation de la dette risquée}

La présentation en termes optionnels des termes de financement permet l'utilisation de la théorie des options pour en déterminer leur valeur. C'est cette évaluation des capitaux propres et de la dette risquée par les modèles d'options que nous allons voir.

Nous présenterons, d'abord, le principe d'évaluation de la dette risquée et, conjointement, de la valeur des capitaux propres. Nous développerons, ensuite, une application chiffrée par application du modèle de Black et Scholes (1973) dans le cadre de l'entreprise simulée en section 1.

\section{Les déterminants de la valeur \\ des sources de financement}

Jensen et Meckling (1976) sont parmi les premiers auteurs à avoir utilisé les modèles d'évaluation d'options, notamment le modèle de Black et Scholes (1973), pour analyser la valeur des capitaux propres de l'entreprise selon la valeur de remboursement de la dette'. Parmi d'autres auteurs précurseurs de cette démarche, on peut aussi citer: Myers (1977) qui analyse la dépendance de la décision d'investissement par rapport à la décision de financement, Geske (1977) qui est à l'origine de la notion "d'option composée" pour l'analyse de la structure financière de l'entreprise dans des situations complexes.

1L'idée que l'action ordinaire d'une entreprise peut être assimilée à une option d'achat a été proposée par Black et Scholes eux-mémes. 
Reconsidérons la situation de l'entreprise simulée dans la précédente section. On a vu que l'on peut définir les capitaux propres comme une option d'achat sur l'actif de la société, l'option devant être exercée à l'échéance de la dette (confondue avec l'échéance du projet) au prix d'exercice de cette dernière. En tant qu'option de type "option européenne" les capitaux propres peuvent être évalués à partir notamment du modèle de Black et Scholes. La valeur des fonds propres va ainsi dépendre des cinq variables bien connues que sont : la valeur de l'actif économique, le prix d'exercice ou valeur de remboursement de la dette, l'échéance, le taux d'intérêt sans risque et, enfin, la variance de la valeur de l'actif, assimilable, ici, à la variance du taux de rentabilité économiquel.

La valeur de la dette risquée apparaît déterminée par le taux sans risque et par la valeur de l'option de vente vendue par les créanciers aux actionnaires. Elle est précisément égale à la valeur de la dette non risquée diminuée de la valeur de f'option de vente sur l'actif de l'entreprise.

Soit :

$$
\begin{aligned}
& D_{0}=D_{0}^{\prime}-P_{0} \\
& D_{0}=D_{n}\left(1+R_{F}\right)^{-n}-P_{0} \\
& D_{0}=D_{n}\left(1+R_{i}\right)^{-n}
\end{aligned}
$$

c'est-à-dire :

valeur actuelle de la dette risquée

$=$ valeur actuelle de la dette sans risque

- valeur de l'option de vente sur l'actif

La valeur du "put" représente la valeur économique de la responsabilité limitée de f'actionnaire, comme nous l'avons vu : elle est donc le prix à payer pour le risque de faillite de l'entreprise. Autrement dit, la valeur de l'option de vente est la valeur de l'option de défaillance qu'acceptent de porter les créanciers.

A partir des modèles d'évaluation des options, on peut dégager les variables qui influencent la valeur de l'optlon de défallance ou prime de risque de la dette.

L'option de défaillance croît avec la valeur de remboursement de la dette, l'éloignement de l'échéance, ainsi que l'augmentation du risque économique mesuré par la variance du prix de l'actif. En revanche l'option de défaillance diminue si la valeur de l'actif augmente ainsi que le taux d'intérêt sans risque.

\footnotetext{
1 On admet que le prix de l'actif est équivalent à sa valeur de marché ; autrement dit, le prix de l'actif est la valeur actualisée des flux financiers qưil génère. En conséquence, la variance de la valeur de l'actif peut être approximée par la variance du taux de rentabilité économique attendu de cet actif.
} 
L'évaluation de l'option de défaillance et de la dette risquée apparaît, ainsi, comme un des apports importants de l'analyse en termes d'option. De même, il devient possible d'analyser la structure financière de l'entreprise en termes d'équilibre entre actionnaires et créanciers ; la simulation chiffrée ci-après en donne l'illustration.

\section{Une simulation chiffrée}

Les conditions de la simulation sont celles qui sont fixées dans la section 1. Elles correspondent aux hypothèses sous-jacentes à l'application du modèle d'arbitrage de Black et Scholes pour l'évaluation d'options. Ces hypothèses sọnt : les marchés sont parfaits, les options sont exercées à l'échéance (option européenne), le taux d'intérêt sans risque est constant, il n'y a pas de distribution de dividendes, la valeur de l'actif économique suit une loi log- normale, par ailleurs, it n'est pas tenu compte des impôts et coûts de transaction.

Soit les données numérique retenues:

- $V_{0}=125$ : montant de l'investissement. Ce montant est aussi la valeur de marché de l'actif, en $t=0$. On suppose qu'il n'y a aucun écart entre la valeur de marché de l'actif et le prix auquel cet actif se négocie sur le marché.

- La durée du projet est de 1 an, l'échéance des options $(t=1)$ est donc de 365 jours.

- $\mathrm{R}_{\theta}=20 \%$ : taux de rentabilité économique requis compte tenu du risque du projet. Par ailleurs, le taux effectif attendu est équivalent au taux requis par le marché1.

- $V_{n}=150$ : valeur attendue de l'actif en $t=1, V_{n}=V_{0}\left(1+R_{e}\right)$.

- La variance, $\sigma^{2}$, du taux de rentabilité est de 0,0256 , soit un écart-type de $16 \% 2$.

- Le taux d'intérêt sans risque est de $\mathrm{R}_{\mathrm{F}}=10 \%$, soit un taux en continu de $9,531 \%$.

\footnotetext{
${ }^{1} \mathrm{~V} n$ et Re n'interviennent pas dans l'évaluation des options, mais leurs prévisions contribuent à l'analyse de la formation de l'équilibre financier de l'entreprise.

2Variance de Re - variance de la valeur de Pactif.
} 
Par ailleurs, il faut choisir a priori une valeur de remboursement de la dette, autrement dit, un prix d'exercice des options. Comme l'objet de la simulation est d'expliquer la détermination de la prime de risque de la dette, d'une part, et la formation de la structure financière, d'autre part, il convient de poser un ensemble hypothétique de valeurs de remboursement de la dette. Les valeurs choisies varient entre 150 et 66 , elles sont contenues dans la première colonne du tableau de résultat de la simulation (tableau 1).

Le tableau 1 est construit par application de la formule de Black et Scholes dont les équations sont rappelées en annexe.

Ce tableau retrace pour chacune des valeurs de remboursement de la dette ou prix d'exercice des options $\left(D_{n}\right)$ :

- la valeur de l'option d'achat, c'est-à-dire des capitaux propres $\left(C_{0}\right)$,

- la valeur de l'option de vente ou valeur de la prime de défaillance $\left(P_{0}\right)$,

- la valeur d'émission de la dette $\left(D_{0}\right)$, valeur des ressources financières mises à la disposition de l'actionnaire pour financer le projet,

- le taux risqué de la dette $\left(R_{i}\right)$, calculé à partir des valeurs de remboursement et d'émission de la dette,

- la structure financière de l'entreprise correspondant au rapport dette/capitaux propres ; en complément, le rapport dette/(capitaux propres + dette) a été calculé. 
TABLEAU 1*

Montants des varlables de financement du projet selon le montant de remboursement prévu de la dette

\begin{tabular}{|c|c|c|c|c|c|c|}
\hline$D_{n}$ & $C_{0}$ & $P_{0}$ & $D_{0}$ & $R$ & ${ }^{D_{0} /} C_{0}$ & ${ }^{D_{\alpha}}{ }_{D_{0}}+C_{0}$ \\
\hline $\begin{array}{r}150 \\
132 \\
121 \\
110 \\
99 \\
88 \\
86 \\
85 \\
84 \\
77 \\
66\end{array}$ & $\begin{array}{r}3,85 \\
10,56 \\
17,26 \\
25,66 \\
35,12 \\
45,01 \\
46,83 \\
47,73 \\
48,64 \\
55,00 \\
65,00\end{array}$ & $\begin{array}{r}15,21 \\
5,56 \\
2,26 \\
0,66 \\
0,12 \\
0,01 \\
0,01 \\
0,01 \\
0,00 \\
0,00 \\
0,00\end{array}$ & $\begin{array}{r}121,14 \\
114,44 \\
107,74 \\
99,34 \\
89,88 \\
79,99 \\
78,17 \\
77,27 \\
76,36 \\
70,00 \\
60,00\end{array}$ & $\begin{array}{l}23,82 \% \\
15,34 \% \\
12,31 \% \\
10,73 \% \\
10,15 \% \\
10,01 \% \\
10,01 \% \\
10,01 \% \\
10,00 \% \\
10,00 \% \\
10,00 \%\end{array}$ & $\begin{array}{r}31,46 \\
10,84 \\
6,24 \\
3,87 \\
2,55 \\
1,78 \\
1,67 \\
1,62 \\
1,57 \\
1,27 \\
0,92\end{array}$ & $\begin{array}{l}0,97 \\
0,92 \\
0,86 \\
0,79 \\
0,72 \\
0,64 \\
0,63 \\
0,62 \\
0,61 \\
0,56 \\
0,48\end{array}$ \\
\hline & $\begin{array}{l}\text { e choisie ap } \\
\text { aleur de rem } \\
\text { es calcultes } \\
\text { aleur des ca } \\
\text { alour de l'op } \\
\text { aleur de ta } \\
\text { ux de la det } \\
\text { = structure }\end{array}$ & $\begin{array}{l}\text { ment de la } \\
\text { ropres en } \\
\text { vente en } t= \\
\text { to }=0 \\
\text { te }\end{array}$ & & & - & \\
\hline
\end{tabular}

Selon les prédictions du modèle on observe :

1. La prime de risque de la dette, représentée par la valeur de l'option de vente, diminue lorsque le volume de la dette dans le financement du projet diminue : en effet, avec la baisse de la part relative de l'endettement, le risque de défaillance pour les créanciers se réduit, entraînant à la baisse la prime de risque qui doit être payée par l'actionnaire. En conséquence, le taux de la dette risquée diminue lui aussi. 
Le taux d'intérêt de la dette tombe au niveau du taux d'intérêt hors risque (10\%), pour un montant de remboursement de la dette voisin de 84 (c'est-àcire une valeur d'émission de 76,36). Ce montant de la dette correspond de fait à la valeur minimum que peut atteindre la valeur de l'actif à l'échéance : compte tenu de la variance du taux de rentabilité économique prévue, la probabilité d'avoir une valeur de l'actif en $t=1$ inférieure à 84 est quasi égale à zéro. En conséquence cette valeur de remboursement de la dette, de 84 , est aussi le seuil en deça duquel les créanciers ne courent plus aucun risque de nonrecouvrement de leur créance. A ce seuil correspond un financement par dette du projet de $61 \%$.

2. II existe deux cas de figure type dans la structure financière de l'entreprise : l'une pour laquelle le risque du projet financé est entièrement porté par l'actionnaire, l'autre dans laquelle il y a partage entre actionnaires et créanciers du risque d'activité.

Si le montant de la dette reste en deça de $61 \%$ de l'investissement, le risque de non-recouvrement pour les créanciers est quasi nul, ils reçoivent le taux sans risque ; les actionnaires supportent l'intégralité du risque d'activité.

Si le montant de la dette devient supérieur à $61 \%$ de l'investissement, les créanciers prennent un risque de non-recouvrement compensé par la hausse des taux. Les créanciers doivent alors implicitement accepter de prendre une part du risque lié au projet, cette part augmentant avec la hausse du volume relatif de la dette.

Le seuil de séparation, ici de $61 \%$, entre les deux types de structures financières est influencé par le taux de rentabilité du projet et la variance de ce dernier : il peut augmenter, et avec lui la part du financement par de la dette non risquée, si le taux de rentabilité augmente et si la variance de ce taux diminue, il diminuera en cas d'évolution inverse de ces deux mêmes variables.

Le problème de la détermination de la structure financière étant posé, il convient d'examiner les conditions de sa formation. 


\section{La formation de la structure financière}

Au regard de la théorie des options la formation de la structure financière de l'entreprise dépend du partage éventuel du risque entre actionnaires ef créanciers (31). Elle dépend ensuite du risque du projet financé (32). Enfin, quels peuvent être la nature et le rôle des garanties que peuvent exiger les créanciers ? (33).

\section{Structure financière et partage du risque}

Toutes les structures financières virtuelles qui peuvent être évaluées à partir des modèles d'évaluation d'options sont des structures indépendantes des préférences des investisseurs face au risque. En effet, la méthode d'évaluation d'options prend appui uniquement sur un raisonnement d'arbitrage et la possibilité qu'il y a de dupliquer l'option.

En conséquence, les structures financières pour lesquelles la valeur de l'option de défaillance est positive ne sont possibles, de fait, que si les créanciers acceptent de prendre le risque correspondant. Se pose, donc, un problème de partage du risque entre actionnaires et créanciers.

Ainsi, selon que les créanciers acceptent ou pas de prendre part au risque on retrouve nos deux cas de figure de structures financières sur lesquelles peut se fixer le choix d'une structure.

Si les créanciers n'acceptent pas de prendre une part du risque, ils imposent un seuil maximum à la valeur du rapport "dette/investissement". Ce sont, en définitive, les créanciers qui fixent la structure financière de l'entreprise. Dans la simulation chiffrée cidessus nous avons observé que le rapport se fixe à $61 \%$.

$\mathrm{Si}$, en revanche, les créanciers acceptent de participer au risque du projet, ils vont accepter des ratios d'endettement plus élevés (supérieurs à $61 \%$ dans la simulation), le financement du projet par dette augmente. La dette sera émise à un taux incluant une prime de risque donnée par la valeur de l'option de défaillance. Plus l'option de défaillance augmentera avec l'augmentation de l'endettement, plus la prime sera forte ainsi que le taux d'intérêt de la dette. Dans cette hypothèse d'un partage du risque, la question demeure de savoir jusqu'à quel montant de risque les créanciers accepteront de s'engager. La réponse implique la mesure de l'attitude des créanciers envers le risque. 1

\footnotetext{
${ }^{1}$ Ce qui suppose la connaissance de la fonction d'utilitié.
} 
L'accroissement de la variété dans l'émission des dettes financières peut être interprété comme une réponse à l'existence de la variété des créanciers selon leurs attitudes face au risque : selon une aversion plus ou moins forte au risque, le niveau de risque accepté étant, toutes choses égales par ailleurs, plus ou moins élevé. Par exemple, le succès des "Junks-bonds", titres de créance relativement risqués par rapport à d'autres qui le sont moins, illustre bien l'existence de situations dans lesquelles les créanciers acceptent une part relativement élevée de risque.

Ainsi, et en conclusion, la structure financière de l'entreprise paraît sinon déterminée du moins influencée par les créanciers selon qu'ils acceptent, compte tenu de leur attitude face au risque, de participer à la prise de risque et, si oui, selon la part plus ou moins grande qu'ils admettent. II y a là un résultat qui converge avec les conclusions des recherches relatives à l'économie des droits contingents entre agents qui sont "risk-adverse". Hirshleifer et Riley (1979), par exemple, démontrent, en effet, qu'il est possible de déterminer des contrats avec partage du risque dans l'échange de droits contingents.

Pareille conclusion ne tient pas compte, cependant, des conflits possibles d'intérêts entre actionnaire et créancier et de l'asymétrie probable d'information entre ces deux agents au détriment généralement des créanciers. Or, ainsi que le démontre la théorie de l'agence [Jensen et Meckling (1976), Myers (977), Smith et Warner (1979), GallaisHamonno et Mourgues (1989), Mourgues (1990)], il existe des problèmes d'agence qui influencent sensiblement les décisions financières prises par les agents et particulièrement le financement par endettement.

\section{Structure financière et \\ risque du projet d'investissement}

Pour un comportement donné des créanciers, la structure financière de l'entreprise apparaît dépendante du risque du projet d'investissement financé.

En effet, lorsque le risque d'activité augmente, la valeur des options, aussi bien d'achat que de vente, sur l'actif de l'entreprise, augmente, toutes choses égales par ailleurs. On trouvera une illustration de ces effets de l'augmentation de la variance du résultat dans le tableau 2 comparativement au tableau 1 : le tableau est construit avec les données de la section 1, seul l'écart-type du taux de rentabilité change, il est de $32 \%$ et non plus de $16 \%$ comme précédemment. 
Montants des varlables de financement du projet de la section 1 , mals avec une valeur de $\sigma=32 \%$

\begin{tabular}{|c|c|c|c|c|c|c|}
\hline$D_{n}$ & $C_{0}$ & $P_{0}$ & $D_{0}$ & $R$ & $D_{0} /$ & $D_{0}$ \\
& & & & & & $C_{0}+C_{0}$ \\
\hline 150 & 11,53 & 22,90 & 113,47 & $32,19 \%$ & 9,84 & 0,91 \\
132 & 18,20 & 13,20 & 106,80 & $23,60 \%$ & 5,87 & 0,85 \\
121 & 23,60 & 8,60 & 101,40 & $19,33 \%$ & 4,30 & 0,81 \\
110 & 30,10 & 5,10 & 94,90 & $15,91 \%$ & 3,15 & 0,76 \\
99 & 37,67 & 2,67 & 87,33 & $13,36 \%$ & 2,32 & 0,70 \\
88 & 46,18 & 1,18 & 78,82 & $11,65 \%$ & 1,71 & 0,63 \\
77 & 55,41 & 0,41 & 69,59 & $10,65 \%$ & 1,26 & 0,56 \\
66 & 65,10 & 0,10 & 59,90 & $10,18 \%$ & 0,92 & 0,48 \\
55 & 75,02 & 0,02 & 49,98 & $10,04 \%$ & 0,67 & 0,40 \\
50 & 79,55 & 0,01 & 45,45 & $10,01 \%$ & 0,57 & 0,36 \\
49 & 80,46 & 0,00 & 44,54 & $10,00 \%$ & 0,55 & 0,36 \\
& & & & & & \\
\hline
\end{tabular}

La lecture du tableau montre bien, comme le veut le modèle d'évaluation d'option, l'augmentation de l'option d'achat et de l'option de vente pour une valeur donnée d'exercice de la dette. Par là même le taux de la dette risquée augmente lui aussi. Par ailleurs, la valeur de l'option de défaillance devient positive pour un ratio "dette/investissement" égal à 0,36, ratio sensiblement inférieur à celui obtenu pour un écart-type de $16 \%$.

En conséquence, pour un même montant de risque supporté par les créanciers, le montant des capitaux propres s'accroît. Ceci revient à admettre que la valeur des capitaux propres augmente lorsque l'écart-type du taux de rentabilité de l'actif augmente. Cette relation mérite d'être commentée.

Du point de vue de la théorie des options cette relation étant contenue dans le modèle d'évaluation lui-même, elle n'appelle aucune explication : la relation est donnée a priori. En revanche, du point de vue du modèle d'évaluation des actifs financiers, il est nécessaire de s'interroger sur le bien-fondé de cette relation. C'est ce qu'ont fait certains auteurs, dont Galai et Masulis (1976) et Hsia (1981). Ces auteurs démontrent, sous certaines hypothèses, qu'il y a bien convergence entre les deux modèles d'évaluation (MEDAF et modèle d'évaluation d'option) et que la valeur des capitaux propres de l'entreprise doit augmenter lorsque la variance des résultats de l'actif financé augmente. La relation, pas du tout évidente, provient du fait que l'accroissement de la variance accroit la probabilité de résultat élevé, ce qui favorise la 
valeur des capitaux propres. En revanche, dans l'hypothèse d'une issue défavorable l'actionnaire peut abandonner les actifs, sa responsabilité étant limitée.

En définitive, lorsque le risque d'un projet augmente, le sous-ensemble de structures financières ne présentant aucun risque pour le créancier se réduit. A l'inverse le sousensemble de structures financières risquées, toujours du point de vue du créancier, s'accroît. Dans l'hypothèse d'un risque élevé du projet financé le sous-ensemble de dette non risquée s'amenuise, il peut devenir nul au-delà d'un certain montant de risque d'activité.

\section{3. Les garanties prises par les créanciers}

Ainsi que l'indique Charreaux (1989), l'approche en termes d'option du financement de l'entreprise permet l'analyse des garanties prises par les créanciers.

L'octroi de garanties permet de réduire le risque de défaillance pour les créanciers. En contrepartie le taux exigé par les créanciers peut être réduit en conséquence. Tout se passe, alors, comme si la valeur de l'option de défaillance, c'est-à-dire la valeur de l'option de vente, était payée de manière autonome et non plus incluse dans la valeur d'émission de la dette pour une valeur de remboursement donnée.

Reconsidérons l'équation suivante établie dans la section 1 :

valeur de l'option d'achat

+ valeur de la dette non risquée

= valeur de l'actif de l'entreprise

+ valeur de l'option de vente.

On a vu que cette équation pouvait être réécrite sous la forme :

valeur de l'option d'achat

+ valeur de la dette risquée

$=$ valeur de l'actif de l'entreprise.

Cette équation, représentative du bilan de l'entreprise, implique que la valeur de l'option de défaillance s'impute sur la valeur de la dette.

Si l'on suppose maintenant qu'il existe une garantie prise par les créanciers et payée par l'actionnaire, garantie qui couvre la valeur de l'option de vente, la dette peut être émise au taux sans risque. La garantie peut être versée à un tiers qui joue le rôle d'assureur auprès des créanciers. Elle peut être versée par un tiers (l'Etat par exempie) lorsque ce dernier souhaite favoriser le financement de certaines activités. Elle peut prendre la forme d'un valeur sur les actifs existants ${ }^{1}$; ce qui explique l'exigence des créanciers qu'il existe une valeur minimum de l'actif net de l'entreprise.

1Valeur non gagée pour le remboursement d'autres créances. 
II n'y a pas, cependant, une équivalence parfaite entre la prime de défaillance lorsque celle-ci est incluse dans la valeur d'émission de la dette et le coût de la garantie demandée. La prime incluse dans la valeur de remboursement de la dette correspond à un paiement différé de l'option de vente : tout se passe comme si les créanciers faisaient crédit à l'actionnaire pour le paiement de l'option de vente. Tandis que la garantie qui peut être demandée pour l'option de défaillance peut être payée à f'émission de la dette. Cela dit, fondamentalement, le coat de la garantle exigée par les créanclers devralt étre déterminé par la valeur de l'option de défalllance.

Mais là encore la conclusion sur le rôle et la valeur de la garantie ne vaut que si l'on ne tient pas compte des problèmes d'agence pouvant exister entre actionnaires et créanciers. Or il peut y avoir une information asymétrique entre les deux catégories d'agents. II peut se faire que les actionnaires soient tentés de ne pas réaliser le projet qui est à la base de l'accord de financement et choisissent un projet concurrent plus risqué, sachant que le projet plus risqué favorise leurs intérêts au détriment de ceux des créanciers [Jensen et Meckling (1976)].

Lorsqu'on introduit le problème d'agence entre actionnaires-créanciers, la garantie revét un second aspect : elle devient un instrument de prévention et de dissuasion que le créancier peut utiliser pour prévenir les comportements de l'actionnaire qui lui sont défavorables [Stulz et Johnson (1985), Mourgues (1990)].

Deux remarques s'imposent pour terminer. Toutes deux ayant trait à la position du créancier dans la relation contractuelle actionnaires-créanciers.

En premier lieu, selon la théorie des options, les créanciers sont décrits comme des vendeurs d'une option de vente. Cette position est une position irréductiblement spéculative : si les créanciers prennent part à la prise de risque, c'est dire qu'ils prennent une position qui joue à la hausse la valeur de l'actif économique de l'entreprise, autrement dit, ils jouent la réussite du projet.

En second lieu, la théorie des options donne une vision quelque peu paradoxale par rapport à lanalyse traditionnelle du financement qui a toujours décrit la position des créanciers comme moins risquée par rapport à celle de l'actionnaire. En effet, en théorie, si la valeur de l'actif à l'échéance est inférieure à la valeur de remboursement de la dette, le risque lié au "put" vendu est supérieur à celui du "call". Autrement dit, la théorie des options révèle des positions de créanciers très risquées, notamment lorsque la part relative de la dette devient importante compte tenu du risque d'activité du projet. En cela, le risque des créanciers peut être supérieur au risque de l'actionnaire. En revanche, si le montant du financement par dette reste réduit, le créancier peut avoir une position sans risque, encore faut-il, cependant, que le risque du projet permette de dégager des structures de financement non risquées pour les créanciers. 


\section{- Conclusion}

L'analyse du financement de l'entreprise à partir de la théorie des options est riche d'un double apport.

D'abord, elle permet d'évaluer la valeur de la dette risquée et par là même de déterminer la prime de risque requise en fonction de la valeur de l'option de défaillance. Conjointement la valeur de cette prime de risque reflète la valeur de la responsabilité limitée des actionnaires, ces derniers détiennent en achetant une option de. vente aux créanciers la faculté d'abandonner l'actif de la société si sa valeur tombe en deça de la valeur de remboursement de la dette.

Ensuite, elle permet d'analyser la formation de la structure financière de l'entreprise et de mettre en évidence les facteurs qui influencent l'équilibre qui peut être négocié entre actionnaires et créanciers. Les résultats obtenus ne sont cependant valables que si l'on néglige les conflits d'intérêts entre les agents ainsi que les problèmes posés par l'asymétrie d'information dans la relation contractuelle. 


\section{- Annexe}

Formules d'évaluation de Black et Scholes

1. Evaluation de l'option d'achat: $C_{0}$

$$
C_{0}=V_{0} N(d 1)-D_{n} e^{-R_{F} t} N(d 2)
$$

avec

$$
\begin{aligned}
& d 1=\frac{\left[L_{n}\left(\frac{V_{0}}{D_{n}}\right)+\left(R_{F}+\frac{1}{2} \sigma^{2}\right) t\right]}{\sqrt{\sigma^{2} t}} \\
& d 2=d 1-\sigma \sqrt{t}
\end{aligned}
$$

2. Evaluation de l'optlon de vente: $P_{0}$

par la relation de périté qui unit le prix du "put" à celui du "call"

$$
P O=C O-V O+D_{n} e^{-R_{F} t}
$$




\section{Bibliographie}

" BLACK F. et SCHOLES M. (1973) : "The Pricing of Options and Corporate Liabilities", Journal of Political Economy, vol. 81.

- CharreauX G. (1989) : Gestion financière, Ed. LITEC, Paris, $2^{e}$ édition.

- Cobbaut R. (1987) : Théorie financière, Ed. Economica, Paris.

- Galais D. et MASUlis R.W. (1976) : «The Option Price Model and the Risk Factor of Stock", Journal of Financial Economics, vol. 3.

- GAllais-HamonNo G. et MOURGues N. (1989) : “L'organisation et les décisions financières de l'entreprise selon la théorie de l'agence : une synthèse de la littérature", Revue d'Economie Financière, n 10.

- GESKE R. (1977): “The Valuation of Corporate Liabilities as Compound Options", Journal of Financial and Quantitative Analysis, vol. 12.

- HIRSHLEIFER J. et RILEY J.G. (1979) : "The Analytics of Incertainty and Information : An Exposition Survey", Journal of Economic Literature, vol. XVII.

- JENSEN M.C. et MECKLING W.H. (1976) : «Theory of the Firm : Managerial Behaviour, Agency Costs and Ownership Structure", Journal of Financial Economics, vol. 3.

- MOURgues $N_{i}$ (1990) : "Les apports de la théorie de l'agence à l'analyse financière externe", Actes du Congrès de l'Association Française de Comptabilité, Saint-Maur, 3-5 mai 1990.

- MYERS S.C. (1977): “Determinants of Corporate Borrowing", Journal of Financial Economics, $n^{\circ} 5$.

- SMITH C.W. et WARNER J.B. (1979): “On Financial Contracting: An Analysis of Bond Convenants", Journal of Financial Economics, vol. 7.

. Stulz R. et Johnson H. (1985) : “An Analysis of Secured Debt», Journal of Financial Economics, vol. 14. 